\title{
Colaboração Através das Alianças e seu Impacto no Desempenho Operacional
}

\author{
Antonio Carlos Domenek ${ }^{1}$ \\ Roberto Giro Moori ${ }^{2}$ \\ Moises Ari Zilber ${ }^{3}$
}

\begin{abstract}
Resumo
O estudo buscou entender a colaboração na cadeia de suprimentos através das alianças de longo prazo e seu impacto no desempenho da empresa compradora. A pesquisa foi dividida em duas fases. Na primeira, de natureza descritiva, desenvolveu-se um questionário estruturado, fundamentado em autores da cadeia de suprimentos e alianças de longo prazo. A segunda fase objetivou aprofundar as percepções sobre colaboração, comparando com os resultados da primeira fase. Foram realizadas entrevistas em profundidade com gerentes de compras responsáveis pela aquisição de materiais e serviços. Os resultados evidenciaram que a redução de custo, o atendimento ao prazo de entrega e a capacidade de resposta são fatores relevantes para o desempenho. O estudo mostrou a importância da colaboração por meio das alianças de longo prazo e seus resultados em empresas instaladas no Brasil.
\end{abstract}

Palavras chave: Cadeia de Suprimentos; Colaboração; Alianças de Longo Prazo; Desempenho Operacional.

\section{Collaboration Through Alliances and its Impact on Operational Performance}

\begin{abstract}
The study aimed to understand the collaboration in the supply chain due long term alliances and its impact on the performance of the purchasing company. A study was carried out in two phases. A descriptive study was developed in the first phase based on authors of supply chain management and long term alliances. A second phase was conducted in order to understand more deeply the perceptions of collaboration and compare with the results of the first phase.
\end{abstract}

\footnotetext{
${ }^{1}$ Engenheiro Mecânico pela Fundação Armando Alvares Penteado(1986). Mestrado em Administração de Empresas (2003) com o tema suprimentos e compras, do Programa de Pós Graduação Stricto Sensu em Administração de Empresas da Universidade Presbiteriana Mackenzie. Atualmente é doutorando em Administração de Empresas na Universidade Presbiteriana Mackenzie. domenek@terra.com.br

${ }^{2}$ Engenheiro mecânico pela Universidade Estadual Paulista Júlio de Mesquita Filho (1980), mestrado em Engenharia (Engenharia de Produção) pela Universidade de São Paulo (1989), doutorado em Engenharia (Engenharia de Produção) pela Universidade de São Paulo (2003), pós doutorado em Administração pela University of Bath, Inglaterra (2003) e em Logística e Engenharia da Informação pela Tokyo University of Marine Science and Techology, Japão (2010). Professor titular da Universidade Presbiteriana Mackenzie e desde 1999 é professor do programa de pós graduação strictu sensu em Administração de Empresas da Universidade Presbiteriana Mackenzie. rgmoori@mackenzie.br
}

${ }^{3}$ Economista pela Faculdade de Economia, Administração e Contabilidade da USP. Doutor e Mestre em Administração pelo Programa de Pós Graduação em Administração pela FEA/USP. Curso de Especialização na França, BEICIP - Bureau dÉtudes Industrielles et du Petrole. Professor Doutor da área de Mestrado e Doutorado em Administração da Universidade Presbiteriana Mackenzie. Exerceu o cargo de Decano (pró Reitor) de Pesquisa e Pós Graduação da Universidade Presbiteriana Mackenzie no período de abril/2011 a maio/2014 quando também presidiu a Câmara de Pesquisa e Pós Graduação da Universidade Presbiteriana Mackenzie. moises.zilber@mackenzie.br 
Interviews were conducted with purchasing managers responsible for procurement of materials and services. The results showed that cost reduction, compliance with delivery time and responsiveness were identified as important factors for the performance of the purchasing company. The study showed the importance of collaboration through long-term alliances and their results in the companies installed in Brazil.

Key words: Supply Chain; Collaboration; Long Term Alliances; Operational Performance.

\section{Introdução}

A Cadeia de Suprimentos (CS) contempla todas as atividades relacionadas ao movimento e a transformação de matérias-primas até produtos acabados, dos fornecedores de produtos primários, passando pelas diversas etapas de manufatura até chegar ao produto e usuário final (KRAUSE; PAGELL; CURKOVIC, 2001) e envolve um constante fluxo de informações, produtos e recursos entre os seus membros, que podem ter diferentes processos e interações nos diversos estágios da cadeia (CHOPRA; MEINDL, 2001).

Nas décadas passadas, houve uma necessidade das empresas olharem para fora de suas organizações buscando oportunidades de colaboração com parceiros para assegurar que a cadeia de suprimentos é dinâmica e responde rapidamente as necessidades dos clientes (CAO; ZHANG, 2011).

$\mathrm{Na}$ busca para se manterem competitivas as organizações devem reconhecer a importância da cadeia de suprimentos (COOK; HEISER; SENGUPTA, 2011), buscando sinergias na integração de processos (PIRES, 2004) e entre as atividades dos parceiros de negócio (LEEUW; FRANSOO, 2009). O melhor desempenho pode ser caracterizado por um conjunto de fatores dependendo do foco que se deseja dar, assim pode-se buscar a melhoria de qualidade (DAVIS; AQUILANO; CHASE, 2001), a redução de custos (CHRISTOPHER, 2001) ou a melhor utilização de um determinado ativo industrial (ZSIDISIN; ELLRAM, 2001). Neste contexto, as empresas que buscam a colaboração (FAWCETT; MAGNAN; MCCARTER, 2008), através das alianças de longo prazo com seus principais fornecedores (LEENDERS; FEARON, 1997; MONCZKA et al., 1998), teriam maior agilidade no atendimento de suas necessidades de materiais e serviços (PAULRAJ; CHEN, 2007), identificada no estudo como a maior capacidade de resposta do fornecedor (SQUIRE et al., 2009) e no melhor atendimento ao prazo de entrega (CHRISTOPHER, 2001), levando a empresa compradora a um desempenho operacional superior, caracterizado pelas reduções de custos (ZSIDISIN; ELLRAM, 2001).

A aliança colaborativa não está associada somente as transações tradicionais de compra e venda (DAVIS; AQUILANO; CHASE, 2001), uma vez que requerem relacionamento contínuo e comprometimento (GENTRY, 1993), com compartilhamento de informações,

ReFAE - Revista da Faculdade de Administração e Economia, v. 9, n. 1, p. 42-62, 2018 
riscos e recompensas (GENTRY, 1993; ZSIDISIN; ELLRAM, 2001). Estas alianças são relações de longo prazo destinadas a aprimorar as capacidades estratégicas e operacionais das empresas participantes, com os benefícios de melhor sincronização da cadeia de suprimentos, redução total de custos, melhoria da qualidade e no tempo de ciclo (MONCZKA et al., 1998). Partindo da premissa que as empresas participantes da pesquisa utilizam como estratégia de suprimentos, a colaboração por alianças de longo prazo, a pesquisa, objeto deste artigo, partiu do seguinte problema: os relacionamentos colaborativos na cadeia de suprimentos das empresas objeto desta pesquisa, decorrentes de alianças de longo prazo, levam ao melhor desempenho operacional da empresa compradora?

A pesquisa se dividiu em duas fases, em uma primeira etapa foram enviados questionários estruturados para oito empresas distintas, classificadas posteriormente como papel e celulose, química/petroquímica e automobilística, com o objetivo de verificar a importância das alianças com fornecedores e os seus reflexos no desempenho operacional da empresa compradora.

$\mathrm{Na}$ segunda fase foram realizadas três entrevistas em profundidade, com gerentes participantes da primeira fase, com experiência superior a cinco anos na função e com responsabilidade sobre a compra de materiais e serviços para as suas organizações, com o objetivo de verificar os resultados obtidos na primeira fase.

O estudo buscou entender se, nas empresas instaladas no Brasil, a colaboração por meio de alianças de longo prazo modifica as relações comerciais entre empresa vendedora e compradora, evoluindo de um caráter apenas tradicional de compra e venda para um relacionamento que agrega outros benefícios e melhorias ao processo. Neste contexto, o estudo é relevante por mostrar, na perspectiva das empresas pesquisadas, a importância das alianças de longo prazo com fornecedores específicos e o seu efeito no desempenho operacional destas organizações.

O estudo foi estruturado contemplando, após a introdução, o referencial teórico sobre gestão da cadeia de suprimentos, colaboração e desempenho operacional. Na sequência foram apresentados os procedimentos metodológicos adotados. Finalizando com a análise dos dados, conclusão final e as recomendações para prosseguimento da pesquisa.

\section{Referencial Teórico}

As empresas buscam tornar a cadeia de suprimentos competitiva, em função do valor que podem adicionar e dos custos que podem ser reduzidos, compreendendo que a competição não será mais entre empresas concorrentes, mas entre as respectivas cadeias de suprimentos

ReFAE - Revista da Faculdade de Administração e Economia, v. 9, n. 1, p. 42-62, 2018 
(CHRISTOPHER, 2001; PAULRAJ; CHEN, 2007), o que implica na necessidade de alinhamento de estratégias e competências dos principais processos de negócios componentes da cadeia de suprimentos (PIRES, 2004).

A gestão competitiva da cadeia de suprimentos deve aumentar o valor (CHRISTOPHER, 2001), ao menor custo, para todos os participantes, perseguindo melhorias em suas atividades internas (BOUNCKEN, 2011), procurando melhorar o desempenho pela eliminação de desperdícios e da utilização eficiente da capacidade do fornecedor, criando uma coordenação na cadeia (PAULRAJ; CHEN, 2007) e nas relações entre fornecedores e compradores a fim de criar valor a um custo menor para a cadeia como um todo (BOUNCKEN, 2011).

O gerenciamento da cadeia de suprimentos (GCS) pode ser entendido como a habilidade de uma empresa em trabalhar com seus fornecedores, buscando obter materiais e serviços com qualidade, prazo e preços competitivos (DAVIS; AQUILANO; CHASE, 2001) e reduzindo as incertezas e riscos que podem afetar os níveis de estoque, os processos e os níveis de serviços ao consumidor final (LEENDERS; FEARON, 1997). É em grande parte a integração de processos, entendendo como integração a condução e o acordo conjunto de atividades entre as empresas dentro da cadeia de suprimentos (FORSLUND; JONSSON, 2009), buscando melhorar tanto o desempenho da organização individualmente como o da cadeia de suprimentos como um todo (BRATIĆ, 2011).

O processo de criação de valor estende para além dos limites da empresa, envolve a integração dos processos e exige que as empresas busquem a integração e a colaboração entre todos os membros da cadeia de suprimentos (GIMENEZ; VENTURA, 2005; VILLENA; REVILLA; CHOI, 2011). Assim, com os fornecedores cada vez mais assumindo papéis estratégicos nas organizações é necessário o desenvolvimento de relações de longo prazo (DAVIS; AQUILANO; CHASE, 2001) que envolvem ligações que utilizam recursos e estruturas das organizações individuais, para se atingir objetivos comuns (SLACK; CHAMBERS; JOHNSTON, 2002).

A gestão colaborativa na cadeia de suprimentos implica na necessidade das empresas formarem alianças (GENTRY, 1993; VIVALDINI; PIRES; SOUZA, 2010), estabelecendo relações de trabalho estreitas e de longo prazo gerando uma interdependência entre as organizações (SOOSAY; HYLAND; FERRER, 2008). Neste contexto a colaboração pelas alianças de longo prazo entre empresas compradoras e fornecedoras estão gradualmente substituindo as relações antagônicas do passado (GENTRY, 1993), tornando o fornecedor não mais uma figura meramente transacional, mas um importante parceiro do negócio (DAVIS; AQUILANO; CHASE, 2001). As verdadeiras alianças requerem obrigatoriamente a 
independência e o compartilhamento de benefícios entre os parceiros (MONCZKA et al., 1998).

Esta nova abordagem proporciona vantagens operacionais para as empresas, compartilhando objetivos comuns, tais como qualidade superior, redução de custos, informações e conhecimentos (GENTRY, 1993), obtendo sinergias entre as organizações (ZSIDISIN; ELLRAM, 2001). Assim, os fornecedores podem prover a organização compradora de muitos dos benefícios de uma integração vertical, como melhor coordenação, melhor utilização dos ativos e respostas rápidas as mudanças de mercado (ZSIDISIN; ELLRAM, 2001).

A colaboração pode ser externa e interna (WIENGARTEN et al., 2010), quando externa a colaboração envolve um relacionamento entre duas ou mais organizações baseada em confiança, abertura, riscos e retornos compartilhados que leva a um melhor desempenho (GENTRY, 1993; ZSIDISIN; ELLRAM, 2001). Assim, maiores níveis de colaboração proporcionam constantes incrementos nos níveis de relacionamento entre as empresas (THOMAS; ESPER; STANK, 2010).

Uma relação de longo prazo com poucos fornecedores tem vantagens, algumas difíceis de quantificar como um potencial para uma maior qualidade (PELEG; LEE; HAUSMAN, 2002), elas são desenhadas para aumentar as capacidades das empresas participantes (MONCZKA et al., 1998).

A decisão de trabalhar com um único ou poucos fornecedores pode estar unicamente relacionada a custos, mesmo nesta situação a decisão ainda pode trazer benefícios por reduzir as incertezas de fornecimento dos materiais e serviços, além de incentivar os fornecedores a praticar preços mais baixos (PELEG; LEE; HAUSMAN, 2002).

As relações estreitas entre fornecedores e compradores criam maiores chances para que as habilidades das partes sejam utilizadas em benefício mútuo (CHRISTOPHER, 2001) e criam o ambiente para o compartilhamento de riscos e recompensas, levando a relacionamentos de longo prazo (PAULRAJ; CHEN, 2007).

A capacidade de trabalhar em conjunto com parceiros tem permitido às empresas integrar e vincular as operações com maior eficácia (SOOSAY; HYLAND; FERRER, 2008), levando as organizações a perseguir processos de melhoria contínua, com o objetivo de produzir produtos e serviços de forma mais eficiente, entregando maior valor a seus clientes (LEENDERS; FEARON, 1997).

A colaboração é importante quando os parceiros percebem que vários benefícios podem ser atingidos como alta qualidade, custo menor, entrega mais rápida, operações eficientes e a coordenação eficaz das atividades (SOOSAY; HYLAND; FERRER, 2008), ela tem um efeito 
importante sobre a relação de interação entre as capacidades externas e o desempenho interno (SQUIRE et al., 2009).

Neste contexto uma das formas em que os relacionamentos de longo prazo, com a consequente maior colaboração entre empresas vendedoras e compradoras, promovam o melhor desempenho da organização compradora está relacionada à maior agilidade da empresa fornecedora em atender as necessidades da empresa compradora.

A agilidade refere-se ao fornecimento pelos parceiros da cadeia de um desempenho superior em tempo de entrega e capacidade de resposta. A capacidade da empresa em responder rapidamente às necessidades dos clientes pode ser uma fonte de persistente vantagem competitiva (PAULRAJ; CHEN, 2007). A organização com foco na agilidade projeta sistemas e procedimentos, com o objetivo de melhorar a velocidade e a confiabilidade de resposta ao cliente (CHRISTOPHER, 2001).

O desenvolvimento de um relacionamento de longo prazo com um número limitado de fornecedores baseado na confiança mútua traz inúmeros benefícios tais como: prazo de entregas mais curtos, entregas confiáveis, entre outros (CHRISTOPHER, 2001), os problemas de qualidade e prazo são transmitidos e resolvidos com maior velocidade (FRANCISCHINI; GURGEL, 2004).

Uma das formas de melhorar a eficiência operacional da cadeia é apressar os fluxos de materiais e de informação dentro da cadeia, com o objetivo de melhor atender aos clientes do produto ou serviço (SLACK; CHAMBERS; JOHNSTON, 2002). A capacidade de resposta está relacionada com a velocidade com que o fornecedor reage a informações da empresa compradora (SQUIRE et al., 2009).

A maior agilidade do fornecedor (menor prazo de entrega e a sua capacidade de resposta) faz com que as incertezas de abastecimento sejam minimizadas permitindo que a organização compradora melhore o seu desempenho operacional. Um processo eficiente é uma medida de sucesso e um fator determinante para a habilidade da empresa em ser lucrativa (CAO; ZHANG, 2011). A implementação integrada da cadeia de suprimentos fornece uma oportunidade estratégica para a criação de competitividade e isso implica no melhor desempenho da empresa (HATANI et al., 2013).

$\mathrm{O}$ aumento da competição, as novas tecnologias e a rápida mudança nos mercados globais forçam as empresas a identificar estratégias para continuar incrementando a sua produtividade (PELEG; LEE; HAUSMAN, 2002), levando as empresas compradoras a procurar estabelecer relacionamentos com seus fornecedores por uma variedade de razões que incluem a redução de custos (ZSIDISIN; ELLRAM, 2001), a sincronização da cadeia (MONCZKA et al., 1998), 
a melhora da qualidade (MONCZKA et al., 1998), entregas mais rápidas e confiáveis (LEENDERS; FEARON, 1997), proporcionando uma melhora na posição competitiva da organização (MONCZKA et al., 1998).

\section{Procedimentos metodológicos}

Para responder ao problema de pesquisa formulado, foi estabelecido como objetivo geral: identificar se nas empresas pesquisadas os relacionamentos colaborativos pelas alianças de longo prazo, entre empresas fornecedoras e compradoras, levam ao melhor desempenho operacional das empresas compradoras. Como objetivos específicos: a) se existe uma diferença de percepção entre os setores industriais pesquisados e b) se nos níveis hierárquicos dos respondentes, dentro da estrutura de compras, a percepção é diferente.

\subsection{Primeira fase da pesquisa}

Nesta fase, o estudo restringiu-se a uma amostra de empresas nacionais e multinacionais de grande porte, instaladas no Brasil. Como sujeitos da pesquisa foram escolhidos compradores, coordenadores e gerentes de compras, com envolvimento na contratação de materiais e serviços. Para a coleta dos dados foi utilizado um questionário estruturado, em que no primeiro bloco procurou-se caracterizar o respondente, em relação ao seu cargo e ao setor de atuação da empresa.

O segundo bloco foi composto de 15 assertivas baseadas no referencial teórico. As assertivas foram constituídas em uma escala ordinal de categorias de discordância/ concordância, do tipo Likert, com seis posições. A opção discordo totalmente recebeu o valor numérico igual a um e a opção concordo totalmente o valor numérico igual a seis. As demais opções da escala receberam valores intermediários entre dois e cinco.

A amostra foi não probabilística onde a seleção dos elementos da população depende, pelo menos em parte, do julgamento do pesquisador e pelo critério de acessibilidade, ou por conveniência (VERGARA, 2000). Neste caso as inferências extraídas da amostra devem ser ressalvadas, em função das limitações deste tipo de abordagem (KERLINGER, 1980).

A estatística descritiva foi utilizada para o tratamento dos dados coletados por meio da distribuição de frequências e porcentagens das respostas obtidas. A distribuição de frequências teve como medidas de tendência central a moda e a mediana, pois elas são indicadas para variáveis obtidas a partir de escala ordinal (SILVER, 2000).

Para verificar as implicações da colaboração pelas alianças de longo prazo entre empresas fornecedoras e compradoras, utilizou-se a análise de frequência das assertivas, modas e 
medianas. Com o objetivo de verificar a existência de diferenças nas respostas entre os setores industriais participantes da pesquisa e do cargo do respondente utilizou-se do teste não paramétrico de Kruskal-Walis que é uma técnica estatística usada para estudar as diferenças entre as médias de duas ou mais populações (MALHOTRA, 2001).

Nesta fase foram enviados 64 questionários para oito empresas dos segmentos de papel e celulose, químico e petroquímico e automobilístico, nos diferentes níveis hierárquicos dentro da estrutura de compras. Retornaram 47 questionários, correspondendo a 73,4\% dos questionários enviados.

\subsection{Segunda fase da pesquisa}

Na segunda fase foram entrevistados três gerentes de compras, com mais de 5 anos na função, e responsáveis pela área de contratação corporativa de materiais e serviços para as respectivas organizações. As empresas onde atuam estes gerentes são líderes em seus mercados, com unidades industriais em diversos estados brasileiros. O objetivo foi o de evidenciar o papel da colaboração por meio das alianças de longo prazo na melhoria do desempenho operacional.

Os dados foram coletados através de entrevista em profundidade utilizando um roteiro préestabelecido. Na aplicação do instrumento de coleta de dados foi definido um protocolo de pesquisa com o objetivo de se estabelecer regras durante a sua aplicação (YIN, 1994).

Finalizada as transcrições das entrevistas foi utilizada a análise de conteúdo com o intuito de obter indicadores para inferir conhecimentos com base na produção e recepção de mensagens (BARDIN, 1977).

\section{Análise dos Dados e Resultados}

\subsection{Primeira Fase}

O perfil dos respondentes foi caracterizado: a) pelo cargo exercido na área de compras e b) pelo setor de atividade das empresas em que estes profissionais atuavam. Em relação ao cargo $21,3 \%$ eram gerentes, $21,3 \%$ coordenadores e $57,4 \%$ compradores, totalizando os 47 questionários respondidos. Em relação às empresas, 46,8\% dos respondentes trabalhavam no setor de papel e celulose, $19,2 \%$ no setor químico e petroquímico e 34,0 \% no setor automobilístico.

Para análise e descrição dos dados utilizou-se a estatística descritiva por meio de frequências e porcentagens das respostas obtidas. A distribuição de frequências, o cálculo da moda e mediana para as assertivas de A1 a A15 são mostrados na Tabela 1. 
Tabela 1: Distribuição de Frequências das Assertivas

\begin{tabular}{|c|c|c|c|c|c|c|c|c|}
\hline \multirow{2}{*}{ ASSERTIVAS } & \multicolumn{3}{|c|}{ DISCORDO } & \multicolumn{3}{|c|}{ CONCORDO } & \multirow{2}{*}{ Moda } & \multirow{2}{*}{ Mediana } \\
\hline & DT & $\mathrm{DM}$ & DP & $\mathrm{CP}$ & $\mathrm{CM}$ & $\mathrm{CT}$ & & \\
\hline $\begin{array}{l}\text { A1- As alianças de longo prazo são uma } \\
\text { estratégia de suprimentos para reduzir custos. }\end{array}$ & 0,0 & 0,0 & 8,5 & 10,6 & 44,7 & 36,2 & 5 & 5,00 \\
\hline $\begin{array}{l}\text { A2- Os fornecedores das alianças de longo prazo } \\
\text { propõem alternativas para redução de custos. }\end{array}$ & 0,0 & 0,0 & 14,9 & 46,8 & 25,5 & 12,8 & 4 & 4,00 \\
\hline $\begin{array}{l}\text { A3- O menor prazo de entrega contribui para a } \\
\text { redução de custos operacionais na minha } \\
\text { organização. }\end{array}$ & 0,0 & 0,0 & 6,4 & 23,4 & 38,3 & 31,9 & 5 & 5,00 \\
\hline $\begin{array}{l}\text { A4- Os fornecedores nas alianças atendem } \\
\text { rapidamente as necessidades da minha } \\
\text { organização. }\end{array}$ & 0,0 & 0,0 & 8,5 & 36,2 & 42,6 & 12,8 & 5 & 5,00 \\
\hline $\begin{array}{l}\text { A5- A redução do custo de compra dos materiais } \\
\text { e serviços foi um dos principais ganhos das } \\
\text { alianças. }\end{array}$ & 0,0 & 0,0 & 6,4 & 34,0 & 29,8 & 29,8 & 4 & 5,00 \\
\hline $\begin{array}{l}\text { A6- Os critérios para medir o sucesso da } \\
\text { colaboração envolvem itens como a redução no } \\
\text { prazo de entrega. }\end{array}$ & 0,0 & 2,1 & 8,5 & 36,2 & 31,9 & 21,3 & 4 & 5,00 \\
\hline $\begin{array}{l}\text { A7- A redução do tempo de resposta do } \\
\text { fornecedor foi um dos principais ganhos da } \\
\text { colaboração. }\end{array}$ & 2,1 & 2,1 & 6,4 & 34,0 & 46,8 & 8,5 & 5 & 5,00 \\
\hline $\begin{array}{l}\text { A8- As alianças de longo prazo são uma } \\
\text { estratégia de suprimentos para reduzir os custos } \\
\text { de transporte. }\end{array}$ & 6,4 & 2,1 & 17,0 & 36,2 & 31,9 & 6,4 & 4 & 4,00 \\
\hline $\begin{array}{l}\text { A9- A redução no prazo de entrega foi um dos } \\
\text { ganhos operacionais das alianças de longo prazo. }\end{array}$ & 0,0 & 4,3 & 10,6 & 29,8 & 42,6 & 12,8 & 5 & 5,00 \\
\hline $\begin{array}{l}\text { A10 - As alianças de longo prazo são uma } \\
\text { estratégia de suprimentos para reduzir os níveis } \\
\text { de estoque. }\end{array}$ & 4,3 & 0,0 & 4,3 & 21,3 & 27,7 & 42,6 & 6 & 5,00 \\
\hline $\begin{array}{l}\text { A11- O menor prazo de resposta do fornecedor } \\
\text { contribui para o melhor desempenho } \\
\text { operacional. }\end{array}$ & 0,0 & 0,0 & 4,3 & 6,4 & 34,0 & 55,3 & 6 & 6,00 \\
\hline $\begin{array}{l}\text { A12- A resposta rápida dos fornecedores é fator } \\
\text { importante nas alianças de longo prazo. }\end{array}$ & 0,0 & 2,1 & 0,0 & 14,9 & 40,4 & 42,6 & 6 & 5,00 \\
\hline $\begin{array}{l}\text { A13- As alianças são uma estratégia de } \\
\text { suprimentos para reduzir as incertezas de } \\
\text { fornecimento. }\end{array}$ & 0,0 & 2,1 & 10,6 & 8,5 & 53,2 & 25,5 & 5 & 5,00 \\
\hline $\begin{array}{l}\text { A14- O prazo de entrega de materiais é fator } \\
\text { importante nas alianças de longo prazo. }\end{array}$ & 0,0 & 2,1 & 0,0 & 17,0 & 44,7 & 36,2 & 5 & 5,00 \\
\hline $\begin{array}{l}\text { A15- O critério principal o sucesso da aliança é a } \\
\text { redução de custos. }\end{array}$ & 0,0 & 0,0 & 0,0 & 8,5 & 46,8 & 44,7 & 5 & 5,00 \\
\hline
\end{tabular}

Nota: DT significa Discordo Totalmente; DM, Discordo Moderadamente; DP, Discordo Parcialmente; CP,

Concordo Parcialmente; CM, Concordo Moderadamente e CT, Concordo Totalmente.

Fonte: os autores

Em relação à assertiva A1 (as alianças de longo prazo são uma estratégia de suprimentos para reduzir custos na minha organização) verificou-se que a maioria dos respondentes, $91,5 \%$ se posicionou na escala de concordância da assertiva com predominância entre os níveis 5 e 6 da escala Likert e 8,5\% discordaram, a mediana das respostas foi de 5,00, o que identificou a existência de concordância em alto grau, mostrando que as alianças de longo prazo com fornecedores geralmente resultaram em benefícios mútuos como a redução geral de custos 
(GENTRY, 1993; MONCZKA et al., 1998; ZSIDISIN; ELLRAM, 2001).

As reduções de custo, que podiam ser obtidas com a adequada gestão da cadeia de suprimentos e a aliança entre empresas compradoras e fornecedoras, estavam relacionadas, entre outras formas, com a redução de estoques (RAJAGOPAL; BERNARD, 1993; ZENZ, 1994), dos custos de transporte (GENTRY, 1993; ZENZ, 1994) e na proposição de alternativas baseadas no conhecimento e competências da empresa fornecedora (GENTRY, 1993; MONCZKA et al., 1998).

Ainda associadas com a redução de custos, as respostas para a assertiva A2 (os fornecedores das alianças de longo prazo propõem alternativas para redução de custos) mostraram também um perfil de concordância, com $85,1 \%$ na escala de concordo e $14,9 \%$ dos respondentes discordando da afirmação. A mediana das respostas se situou em 4,00, com o valor de maior frequência (moda) em 4, o que mostrou a concordância em menor grau quanto a proposição que os fornecedores de longo prazo propõem alternativas para a redução de custos da organização compradora. Outras assertivas relacionadas à redução de custos são a A5 (a redução do custo de compra dos materiais e serviços foi um dos principais ganhos das alianças), A8 (as alianças de longo prazo são uma estratégia de suprimentos para reduzir os custos de transporte) e A10 (as alianças de longo prazo são uma estratégia de suprimentos para reduzir os níveis de estoque) também apresentaram alto nível de concordância, mínima acima de 74,5\%. Em relação à assertiva 8 , a mediana foi de 4,00 e a moda igual a 4, e a distribuição de frequências com a maior porcentagem na escala da discordância entre todas as assertivas, apesar de a maioria das respostas ainda estarem no lado da concordância da escala, o que pode mostrar que para estas empresas, as reduções de custo relacionadas a transporte não foram as mais relevantes ou não foram o foco dos contratos/alianças de longo prazo.

Em resumo: as assertivas relacionadas à redução de custo mostraram que, no entender dos respondentes das oito empresas participantes, este fator era reconhecido como um importante resultado e direcionador do relacionamento colaborativo pelas alianças de longo prazo entre a sua empresa e as empresas fornecedoras.

Em relação à agilidade de atendimento por parte da empresa fornecedora, com o menor prazo de entrega contribuindo para a redução dos custos (CHRISTOPHER, 2001; FRANCISCHINI; GURGEL, 2004), a assertiva A3 mostra forte grau de concordância $(93,6 \%)$ com a afirmação que o menor prazo de entrega contribuiu para a redução de custos operacionais da organização e 6,4\% na escala de discordância, com mediana 5,00 e moda de 5. Ainda em relação a prazo de entrega, as assertivas A6 (os critérios para medir o sucesso da colaboração envolvem itens como a redução no prazo de entrega) e A14 (O prazo de entrega de materiais é

ReFAE - Revista da Faculdade de Administração e Economia, v. 9, n. 1, p. 42-62, 2018 
fator importante nas alianças de longo prazo), também associadas ao prazo, tiveram graus de concordância acima de $85,2 \%$ o que mostra que os respondentes identificam o prazo de entrega, e suas implicações para a eficiência e o desempenho organizacional, como um importante componente dos contratos de longo prazo.

A prontidão de atendimento e a capacidade de resposta rápida as necessidades da empresa compradora (CHRISTOPHER, 2001) foram uma fonte de vantagem competitiva (PAULRAJ; CHEN, 2007) e estavam relacionadas com a velocidade com que o fornecedor reagia a informações da empresa compradora (SQUIRE et al., 2009).

Em relação à prontidão de atendimento e a capacidade de resposta do fornecedor, representado pelas assertivas A4 (os fornecedores nas alianças atendem rapidamente as necessidades da minha organização), A7 (a redução do tempo de resposta do fornecedor foi um dos principais ganhos da colaboração), A11 (o menor prazo de resposta do fornecedor contribuiu para o melhor desempenho operacional) e A12 (a resposta rápida dos fornecedores é fator importante nas alianças de longo prazo) apresentaram respostas às assertivas com grau de concordância, nas empresas pesquisadas, superiores a 89,3\%.

Um fator de destaque foi a assertiva 11, com o maior grau de concordância entre as quatro assertivas referentes ao conceito de atendimento rápido do fornecedor de alianças de longo prazo. Esta assertiva relacionava o menor prazo de resposta do fornecedor com o melhor desempenho operacional, o que pode mostrar que na opinião dos respondentes a maior capacidade de resposta é fator relevante para o melhor desempenho operacional da empresa compradora.

A assertiva 13 era relacionada à redução das incertezas de fornecimento conseguidas por meio das alianças de longo prazo. O grau de concordância foi de 87,2\% com mediana de 5,00 e moda 5, o que mostrou que nas empresas pesquisas estes contratos de longo prazo contribuíam para a redução da incerteza do fornecimento.

Finalizando, as respostas da assertiva A15 (o critério principal o sucesso da aliança é a redução de custos) apresentaram um perfil maior que o das demais respostas, com nível na escala de concordância de $100 \%$, com mediana das respostas em 5,00 e frequência de resposta entre os níveis 5 e 6 da escala. Este nível de concordância nas respostas pode mostrar que a redução de custos e o melhor desempenho operacional foram os principais motivadores quando do estabelecimento de alianças de longo prazo com os fornecedores. As organizações de compras escolhiam as alianças por várias razões, entre elas estavam a redução de custos, a melhora do serviço e a vantagem competitiva (ZSIDISIN; ELLRAM, 2001).

Em resumo: para todas as assertivas, as respostas na faixa de concordância foram maiores que

ReFAE - Revista da Faculdade de Administração e Economia, v. 9, n. 1, p. 42-62, 2018 
na faixa de discordância, com destaque para as assertivas A8 com menor grau de concordância $(74,5 \%)$ e relacionada com a redução de custos no transporte e para a assertiva A15 com o maior grau de concordância (100\%), relacionada com a redução de custos e melhor desempenho operacional como principais indicadores do sucesso de um contrato de longo prazo.

$\mathrm{Na}$ sequência procurou-se verificar se existiam diferenças entre as respostas em função do cargo do respondente conforme são mostrados na Tabela 2.

Tabela 2: Teste não paramétrico de Kruskal-Walis (Diferenças entre cargos)

\begin{tabular}{|c|c|c|c|c|c|c|c|c|c|c|}
\hline \multirow{3}{*}{ Assertivas } & \multirow{2}{*}{\multicolumn{3}{|c|}{ Média }} & \multicolumn{6}{|c|}{ Limite Inferior (LI) e Superior (LS) } & \multirow{3}{*}{$\begin{array}{l}\text { Kruskal-Walis } \\
\text { Significância }\end{array}$} \\
\hline & & & & \multicolumn{3}{|c|}{ LI } & \multicolumn{3}{|c|}{ LS } & \\
\hline & $\mathrm{CP}$ & $\mathrm{CD}$ & $\mathrm{G}$ & $\mathrm{CP}$ & $\mathrm{CD}$ & $\mathrm{G}$ & $\mathrm{CP}$ & $\mathrm{CD}$ & $\mathrm{G}$ & \\
\hline A1 & 5,11 & 5,20 & 4,90 & 4,79 & 4,54 & 4,04 & 5,43 & 5,86 & 5,76 & 0,857 \\
\hline $\mathrm{A} 2$ & 4,37 & 4,50 & 4,20 & 3,96 & 3,97 & 3,54 & 4,77 & 4,88 & 4,86 & 0,636 \\
\hline A3 & 4,70 & 5,50 & 5,10 & 4,36 & 4,99 & 4,39 & 5,05 & 6,01 & 5,81 & 0,039 \\
\hline A4 & 4,63 & 4,80 & 4,30 & 4,30 & 4,35 & 3,62 & 4,96 & 5,25 & 4,98 & 0,396 \\
\hline A5 & 4,96 & 5,00 & 4,30 & 4,59 & 4,33 & 3,71 & 5,33 & 5,67 & 4,89 & 0,117 \\
\hline A6 & 4,48 & 4,90 & 4,70 & 4,05 & 4,37 & 4,91 & 5,04 & 5,43 & 5,38 & 0,508 \\
\hline A7 & 4,44 & 4,60 & 4,40 & 3,99 & 4,10 & 3,90 & 4,90 & 5,10 & 4,90 & 0,859 \\
\hline A8 & 4,00 & 4,50 & 3,70 & 3,46 & 3,99 & 2,94 & 4,54 & 5,01 & 4,46 & 0,216 \\
\hline A9 & 4,33 & 4,80 & 4,60 & 3,91 & 4,35 & 3,83 & 4,76 & 5,25 & 5,37 & 0,372 \\
\hline A10 & 4,89 & 5,40 & 4,70 & 4,33 & 4,80 & 4,02 & 5,45 & 6,00 & 5,38 & 0,260 \\
\hline A11 & 5,41 & 5,50 & 5,30 & 5,11 & 4,80 & 4,71 & 5,70 & 6,20 & 5,89 & 0,645 \\
\hline A12 & 5,19 & 5,40 & 5,10 & 4,80 & 4,90 & 4,57 & 5,57 & 5,90 & 5,63 & 0,655 \\
\hline A13 & 4,89 & 5,00 & 4,80 & 4,49 & 4,42 & 3,99 & 5,29 & 5,58 & 5,61 & 0,964 \\
\hline A14 & 5,15 & 5,20 & 5,00 & 4,77 & 4,64 & 4,52 & 5,52 & 5,76 & 5,48 & 0,685 \\
\hline A15 & 5,59 & 5,30 & 4,80 & 5,37 & 4,82 & 4,50 & 5,82 & 5,78 & 5,10 & 0,003 \\
\hline
\end{tabular}

Nota: CP significa Comprador; CD, Coordenador e; G, Gerente

Fonte: os autores

Na Tabela 2, são mostradas as médias, os limites inferiores e superiores e significância estatística (p) para a diferença entre as médias das amostras significante para $(\alpha \leq 0,05)$ para os cargos de comprador (CP), coordenador (CD) e gerente $(\mathrm{G})$. Com exceção das assertivas A3 e A15, com significância menor que 0,05, não existiram diferenças entre as respostas de compradores, coordenadores e gerentes de compras.

Em relação a assertiva A3, o maior grau de concordância ficou entre os coordenadores e o menor entre os compradores, sugerindo que a função mais operacional da estrutura de compras relacionou em menor intensidade a redução dos custos operacionais com o prazo de entrega. Em relação a assertiva A15, a maior concordância ficou com os compradores e a menor com os gerentes, podendo sugerir que os compradores focam mais na redução de custos imediatos e avaliam o sucesso da aliança por este ponto, enquanto que os gerentes de compras teriam uma visão mais estratégica destas alianças e dos seus resultados. 
Quanto à comparação das médias entre os setores de Papel e Celulose (PC), Químico e Petroquímico (QP) e Automobilístico (AU), os resultados são mostrados na Tabela 3.

Tabela 3: Teste não paramétrico de Kruskal-Walis (Diferenças entre setores)

\begin{tabular}{ccccccccccc}
\hline & & \multirow{3}{*}{ Assertivas } & & \multicolumn{4}{c}{ Média } & & \multicolumn{4}{c}{ LI } & \multicolumn{4}{c}{ LS } & \multirow{2}{*}{\begin{tabular}{c} 
Kruskal-Walis \\
\cline { 8 - 10 }
\end{tabular}} & PC & QP & AU & PC & QP & AU & PC & QP & AU & Significância \\
\hline A1 & 5,18 & 5,67 & 4,63 & 4,92 & 5,28 & 3,98 & 5,44 & 6,05 & 5,27 & 0,038 \\
A2 & 4,36 & 4,44 & 4,31 & 3,94 & 3,89 & 3,81 & 4,79 & 5,00 & 4,82 & 0,939 \\
A3 & 5,00 & 5,22 & 4,75 & 4,59 & 4,71 & 4,22 & 5,41 & 5,73 & 5,28 & 0,512 \\
A4 & 4,73 & 5,22 & 4,06 & 4,45 & 4,58 & 3,65 & 5,01 & 5,86 & 4,47 & 0,002 \\
A5 & 4,95 & 5,56 & 4,25 & 4,56 & 5,15 & 3,79 & 5,35 & 5,96 & 4,71 & 0,002 \\
A6 & 4,77 & 5,00 & 4,19 & 4,44 & 4,23 & 3,57 & 5,11 & 5,77 & 4,81 & 0,095 \\
A7 & 4,77 & 4,89 & 3,81 & 4,50 & 4,29 & 3,19 & 5,04 & 5,49 & 4,43 & 0,009 \\
A8 & 4,41 & 4,44 & 3,31 & 4,11 & 3,22 & 2,65 & 4,70 & 5,67 & 3,98 & 0,008 \\
A9 & 4,64 & 4,89 & 4,06 & 4,29 & 3,99 & 3,50 & 4,99 & 5,79 & 4,63 & 0,058 \\
A10 & 5,36 & 5,56 & 4,06 & 5,01 & 5,00 & 3,27 & 5,71 & 6,11 & 4,85 & 0,002 \\
A11 & 5,50 & 5,78 & 5,06 & 5,17 & 5,44 & 4,57 & 5,83 & 6,12 & 5,56 & 0,075 \\
A12 & 5,32 & 5,78 & 4,75 & 5,03 & 5,44 & 4,18 & 5,60 & 6,12 & 5,32 & 0,013 \\
A13 & 5,14 & 5,44 & 4,25 & 4,82 & 5,04 & 3,62 & 5,45 & 5,85 & 4,88 & 0,008 \\
A14 & 5,27 & 5,33 & 4,81 & 4,99 & 4,95 & 4,19 & 5,55 & 5,72 & 5,43 & 0,394 \\
A15 & 5,41 & 5,67 & 5,13 & 5,19 & 5,28 & 4,70 & 5,63 & 6,05 & 5,55 & 0,177 \\
\hline
\end{tabular}

Nota: PC significa Papel e Celulose; QP, Químico e Petroquímico e AU: Automobilístico

Fonte: os autores

Na Tabela 3, observou-se que em nível de significância $(\alpha \leq 0,05)$ existiram diferenças entre as respostas das assertivas A1, A4, A5, A7, A8, A10, A12 e A13. O fato pode ter sido influenciado pelas respostas de profissionais das empresas classificadas no setor automobilístico, visto que as empresas não eram homogêneas (montadora e autopeças), o que pode ter levado a diferenças de percepção sobre o tema entre os respondentes das empresas.

\subsection{Segunda Fase}

Nesta fase procurou-se verificar as considerações de três profissionais de compras experientes, em relação à colaboração pelas alianças e os seus resultados para a organização compradora, em termos de agilidade e de melhoria no desempenho operacional.

Os entrevistados ressaltaram a diferença entre os contratos ou pedidos de compras das alianças de longo prazo entre empresa compradora e fornecedora, que levava a um maior comprometimento por parte do fornecedor gerando benefícios para ambas as empresas.

As alianças com fornecedores eram de longo prazo (MONCZKA et al., 1998), ofereciam benefícios (PAULRAJ; CHEN, 2007) e reduziam substancialmente as incertezas de custo (PELEG; LEE; HAUSMAN, 2002), não obstante, requeriam um relacionamento baseado em confiança mútua, abertura, riscos e retornos compartilhados (ZSIDISIN; ELLRAM, 2001). As alianças excediam as possíveis contribuições das relações tradicionais (MONCZKA et al., 
1998).

Ficou muito claro pela fala dos entrevistados que as alianças de longo prazo eram ações ou processos diferentes e que levavam a resultados distintos, conforme pode ser observado nas transcrições, mostradas a seguir. Os grifos nas transcrições foram colocados pelos autores para destacar os aspectos importantes em cada fala.

Em relação à distinção dos contratos de fornecimento de materiais e serviços que eram considerados como alianças de longo prazo dos contratos comuns, sendo que neste último caso estariam inclusos os pedidos de compra de curto prazo.

(...) porque nem todos os contratos de fornecimento chegam a ser uma aliança (...). (Entrevistado 1)

(...) fornecedores que tenho alianças estratégicas de longo prazo (...) e também tem os de compras spot (...) (Entrevistado 2)

(...) nós normalmente diferenciamos contratos que são alianças, em relação ao mero contrato de fornecimento. (Entrevistado 1)

(...) é a diferença do fornecedor que é só o spot para o fornecedor de longo prazo (...) você possa a ter essa segurança que só vem com o tempo e as experiências (...) (Entrevistado 3)

Apesar da colaboração entre empresas, os entrevistados ressaltaram a necessidade dos contratos formais como uma forma de garantir o entendimento do acordado entre as partes. Para que fossem observadas todas as medidas que os parceiros se comprometeram era necessária à existência de uma relação contratual (DAS; SEN; SENGUPTA, 1998).

(...) carece de formalização, ele carece de rastreabilidade, ele carece de garantias de parte a parte ao que será realizado ao longo do tempo (...) (Entrevistado 1)

(...) tudo é sempre bem formalizado, através de contratos (Entrevistado 3)

(...) um contrato jurídico específico (...) ele leva em conta as necessidades do nosso cliente e as necessidades da nossa empresa de garantia, seguros, entre outras coisas (...) (Entrevistado 2)

O Entrevistado 1 destacou à necessidade de contratos de longo prazo para ganhos superiores. Para o Entrevistado 2, os relacionamentos de longo prazo levaram a uma possibilidade de ReFAE - Revista da Faculdade de Administração e Economia, v. 9, n. 1, p. 42-62, 2018 
redução de custos para as empresas.

Nós temos (...) contratos iniciais de longo prazo firmados por períodos superiores a 10 anos, após a recuperação do capital do fornecedor foram renovados em bases mutuamente satisfatórias. (Entrevistado 1)

(...) é muito difícil adotar estas soluções, que eu disse, com um ambiente competitivo, com alternâncias de fornecedor, com períodos de contratos muito curtos (...) (Entrevistado 1).

(...) essa colaboração de longo prazo (...) acaba criando essa... confiança entre fornecedor e comprador (...) não adianta negociar desconto (...) você só reduz custo quando você faz algo diferente (Entrevistado 2).

Falando ainda em relação às alianças entre empresas fornecedoras e empresas compradoras, o Entrevistado 1 ressaltou que muitas vezes o relacionamento entre empresa fornecedora e compradora se estendia por um longo tempo, superando em muito os prazos originais contratados. O Entrevistado 3 destacou a figura dos fornecedores estratégicos de longo prazo em que há uma maior abertura de informações, inclusive sobre projetos futuros da empresa. Com os fornecedores cada vez estratégicos era necessário o desenvolvimento de relações de longo prazo, levando muitas vezes a contratos que eram renovados automaticamente (DAVIS; AQUILANO; CHASE, 2001).

(...) surgem alianças que transcendem o período de vigência de um contrato e acabam se tornando sequências de contratos que vão sendo aprimorados ao longo do tempo e garantindo relacionamentos de longo prazo (...) (Entrevistado 1)

(...) são com os fornecedores estratégicos que eu faço a parceria de longo prazo, com ele que eu abro os projetos especiais e para ele que abro para que projeto eu estou trabalhando (...) (Entrevistado 3)

(...) uma verdadeira aliança, quando construída em bases sólidas, ela provavelmente vai transcender o período do contrato original (...) (Entrevistado 1)

Os trechos apresentados acima sinalizaram, em relação as empresas pesquisadas, a natureza e as vantagens da aliança de longo prazo entre fornecedores e compradores, em que o 
relacionamento entre as organizações levava ao comprometimento e a colaboração entre as empresas.

A colaboração nas cadeias de suprimentos era importante (SOOSAY; HYLAND; FERRER, 2008) e tinha um efeito significativo na relação de interação entre as capacidades externas e o desempenho interno (SQUIRE et al., 2009).

As alianças de longo prazo podiam resultar em melhores prazos de entrega e de capacidade de resposta por parte do fornecedor. A capacidade de resposta era a agilidade do fornecedor em atender prontamente as necessidades da empresa compradora.

A capacidade da empresa para responder rapidamente às necessidades dos clientes podia ser uma fonte de persistente vantagem competitiva (PAULRAJ; CHEN, 2007), a organização ágil projetava sistemas e procedimentos, com o objetivo de melhorar a velocidade e a confiabilidade de resposta (CHRISTOPHER, 2001).

Neste contexto, as falas dos entrevistados levaram a visualizar alguns destes compromissos e ganhos nas empresas pesquisadas, incluindo uma postura mais colaborativa entre as empresas compradoras e fornecedoras.

(...) havendo um relacionamento desta natureza cujo valor de parte a parte e percebido como mais alto, isso vai produzir uma maior prontidão, uma maior resposta em relação às necessidades (...) (Entrevistado 1).

(...) relações mais colaborativas impactam no prazo... prazo é uma coisa muito importante (...) esse relacionamento (...) facilita muito o desenvolvimento de soluções em que você possa ganha prazo. (Entrevistado 3)

Ainda em relação à agilidade de atendimento, ela pode ser conseguida pela empresa compradora e entregue pela empresa fornecedora através do avanço da sua linha de produção, com a colocação de etapas do processo ou disponibilização de estoques avançados, assim como as melhorias de logística na cadeia de suprimentos que podiam fazer com que o prazo de entrega fosse minimizado e que a confiabilidade no fornecimento fosse aumentada.

Deste modo, as empresas trabalhavam mais próximas aos seus fornecedores, eliminando seus estoques de proteção e incorporando atividades dos fornecedores dentro das suas próprias instalações (DAVIS; AQUILANO; CHASE, 2001), um menor custo de produção, muitas vezes, necessitava investimentos especializados para o fornecimento (VANDEGRIFT, 1998). 
nas cadeias os prazos, as dificuldades de logística (...). (Entrevistado 1)

(...) uma unidade produtiva que integra totalmente ou uma finalização do produto do fornecedor já próximo da unidade consumidora (...) (Entrevistado 1)

(...) fornecedores trazem uma solução integrada, ou seja, em vez de nós comprarmos todos os componentes separados, hoje eles nos fornecem um sistema. (Entrevistado 3)

(...) temos situações, por exemplo, de fornecedores que atuam aqui dentro. A empresa de montagem que vem e monta um painel dentro de uma estrutura da máquina (...) eu vejo isso como uma gestão colaborativa. (Entrevistado 2)

O melhor desempenho operacional obtido com a redução de custos foi comentado pelos entrevistados, nas suas organizações, por vários ângulos. A melhora da eficiência operacional e com os ganhos obtidos tanto com a redução do preço do produto, melhoria de processos, assim como por melhorias logísticas levavam a um melhor desempenho da organização compradora. A estratégia de contenção de custos necessita da integração entre as empresas e seus fornecedores (RAJAGOPAL; BERNARD, 1993).

(...) estamos falando não só da questão da integração, do prazo de pagamento, da redução de estoque, da garantia de bom custo que são coisas que com certeza vão ser endereçadas na aliança estratégica porque negócio é negócio, não se pode perder o foco no resultado. (Entrevistado 1)

Totalmente, eu diria que a incerteza do fornecimento é um custo e a supressão da incerteza do fornecimento, ela é um valor. (Entrevistado 1)

(...) se o fornecedor conhece as suas necessidades e sabe o que você precisa a gente vai convergir para uma melhoria de processo e uma redução de custo. (Entrevistado 2)

(...) o que todo mundo busca, eficiência, diminuir o seu custo, claro se você vai ser eficiente vai diminuir o que você está... o custo da sua operação (...) (Entrevistado 3)

Em resumo: pelas considerações dos gerentes de compras evidenciou-se que nas organizações os relacionamentos colaborativos pelas alianças de longo prazo ainda necessitavam de formalização através de contratos que podiam ter prazo de duração superior a 10 anos. No 
sentido da colaboração, em algumas situações, estes contratos eram renovados periodicamente em bases mutuamente satisfatórias, trazendo como benefícios a redução de custos, de estoques, prazo de entrega e a prontidão de resposta do fornecedor.

\section{Conclusão e Sugestões para Prosseguimento da Pesquisa}

Em relação ao objetivo principal, verificou-se que o grau de concordância com as assertivas apresentadas, que relacionavam as alianças de longo prazo com a redução de custos, os menores prazos de entrega e a capacidade de resposta do fornecedor, foi de no mínimo 74,5\%, mostrando que, no entender da maioria dos respondentes das oito empresas participantes, estes fatores foram reconhecidos como importantes resultados da colaboração por alianças entre empresa compradora e as empresas fornecedoras, com a totalidade dos respondentes concordando que o critério principal para medir o sucesso da aliança de longo prazo estava relacionado à redução de custos e ao melhor desempenho operacional. O resultado foi confirmado nas entrevistas realizadas com os gerentes de compras que destacaram o papel das alianças de longo prazo nas suas organizações e que estas levavam as empresas a atingir resultados operacionais superiores, considerando além da redução de custos dos materiais adquiridos, a redução dos estoques, a melhoria dos processos logísticos, os melhores prazos de entrega e a maior prontidão de atendimento do fornecedor.

A análise de conteúdo das entrevistas ainda permitiu verificar que nas alianças de longo prazo, nas três empresas em questão, ainda existia a formalização do processo através de contratos que eram em algumas situações renovados em condições mutuamente satisfatórias, o que levava a deduzir que foram vantajosos e trouxeram bons resultados tanto para as empresas fornecedoras como para a empresa compradora.

Com relação aos objetivos específicos: a) se existe uma diferença de percepção entre os diferentes setores industriais pesquisados, verificou-se que apesar do grau de concordância, as percepções são diferentes se comparados os setores de papel e celulose, química e petroquímica e automobilístico e; b) se nos níveis hierárquicos dos respondentes, dentro da estrutura de compras, a percepção é diferente. Em relação a este último objetivo foi possível evidenciar que entre as diferentes empresas pesquisadas o cargo do respondente não influiu na identificação e percepção do impacto das alianças no desempenho operacional da organização.

Limitações do método: o estudo apresentou as seguintes limitações: a) tamanho da amostra ficou aquém do mínimo necessário de 75 respondentes e; b) critério de escolha das empresas foi por acessibilidade. Em razão destas limitações, os resultados obtidos devem ser vistos com

ReFAE - Revista da Faculdade de Administração e Economia, v. 9, n. 1, p. 42-62, 2018 
ressalvas.

Diante do exposto, sugere-se para prosseguimento desta pesquisa: a) ampliar a amostra focando em um setor específico; b) analisar outros setores com característica mais similares em relação ao seu processo produtivo, como por exemplo, produção de ciclo contínuo, produção seriada, produção por encomenda, entre outros e c) verificar se existe diferença dentro do mesmo setor de atividade, entre empresas de grande porte e de pequeno porte.

Por fim, o estudo foi relevante por mostrar a importância da colaboração com fornecedores estratégicos, por alianças de longo prazo, em empresas instaladas no Brasil.

\section{Referências}

BARDIN, L. Análise de Conteúdo. Lisboa: Edições 70, 1977.

BOUNCKEN, R. B. Supply Chain Contingencies: The Effects of Up-Stream Directives on Supplier's Innovation Performance. Engeneering Management Journal, v. 23, n. 4, 2011.

BRATIĆ, D. Achieving a Competitive Advantage by SCM. IBIMA Business Review Journal, v. 2011, p. 1-13, 2011.

CAO, M.; ZHANG, Q. Supply chain collaboration: Impact on collaborative advantage and firm performance. Journal of Operations Management, v. 29, n. 3, p. 163-180, mar. 2011.

CHOPRA, S.; MEINDL, P. Supply Chain Management: Strategy, Planning and Operation. New Hersey: Prentice Hall, 2001.

CHRISTOPHER, M. Logística e Gerenciamento da Cadeia de Suprimentos. São Paulo: Pioneira Thomson Learning, 2001.

COOK, L. S.; HEISER, D. R.; SENGUPTA, K. The moderating effect of supply chain role on the relationship between supply chain practices and performance: An empirical analysis. International Journal of Physical Distribution \& Logistics Management, v. 41, n. 2, p. 104-134, 2011.

DAS, S.; SEN, P. K.; SENGUPTA, S. Impact of strategic alliances on firm valuation. Academy of Management Journal, v. 41, n. 1, p. 27-41, 1998.

DAVIS, M. M.; AQUILANO, N. J.; CHASE, R. B. Fundamentos da Administração da Produção. 3a. ed. Porto Alegre: Bookman, 2001.

FAWCETT, S. E.; MAGNAN, G. M.; MCCARTER, M. W. A Three-Stage Implementation Model for Supply Chain Collaboration. Journal of Business Logistics, v. 29, n. 1, p. 93-112, 2008. 
FORSLUND, H.; JONSSON, P. Obstacles to supply chain integration of the performance management process in buyer-supplier dyads: The buyers' perspective. International Journal of Operations \& Production Management, v. 29, n. 1, p. 77-95, 2009.

FRANCISCHINI, P. G.; GURGEL, F. DO A. Administração de Materiais e do Patrimônio. São Paulo: Pioneira Thomson, 2004.

GENTRY, J. J. Strategic alliances in purchasing: Transportation is the vital link. International Journal of Purchasing and Materials Management, v. 29, n. 3, p. 11, 1993.

GIMENEZ, C.; VENTURA, E. Logistics-production, logistics-marketing and external integration: Their impact on performance. International Journal of Operations \& Production Management, v. 25, n. 1, p. 20-38, 2005.

HATANI, L. et al. The Role of Competitiveness as Mediator for the Relation between Supply Chain Flexibility and Firm Performance. Journal of Management Research, v. 5, n. 1, p. 269-291, 2013.

KERLINGER, F. N. A metodologia da pesquisa em ciências sociais: um tratamento conceitual. 1. ed. São Paulo: EPU, 1980.

KRAUSE, D. R.; PAGELL, M.; CURKOVIC, S. Toward a measure of competitive priorities for purchasing. Journal of Operations Management, v. 19, n. 4, p. 497-512, 2001.

LEENDERS, M. R.; FEARON, H. E. Purchasing and Supply Management. 11 a ed.EUA: Irwin McGraw-Hill, 1997.

LEEUW, S. DE; FRANSOO, J. Drivers of close supply chain collaboration: one size fits all? International Journal of Operations \& Production Management, v. 29, n. 7, p. 720-739, 2009.

MALHOTRA, N. K. Pesquisa de Marketing : uma orientação aplicada. Porto Alegre: Bookman, 2001.

MONCZKA, R. M. et al. Success factors in strategic supplier alliances: The buying company perspective. American Institute for Decision Sciences, v. 29, n. 3, p. 553-577, 1998.

PAULRAJ, A.; CHEN, I. J. Strategic Buyer?Supplier Relationships, Information Technology and External Logistics Integration. The Journal of Supply Chain Management, v. 43, n. 2, p. 2-14, 2007.

PELEG, B.; LEE, H. L.; HAUSMAN, W. H. Short-term e-procurement strategies versus long-term contracts. Production and Operations Management, v. 11, n. 4, p. 458-480, 2002.

PIRES, S. R. I. Gestão da Cadeia de Suprimentos. São Paulo: Atlas, 2004. 
RAJAGOPAL, S.; BERNARD, K. N. Cost Containment Strategies: Challenges for Strategic Purchasing in the 1990s. International Journal of Purchasing and Materials Management, v. 29, n. 1, p. 17-24, 1993.

SILVER, M. Estatística para a administração. São Paulo: Atlas, 2000.

SLACK, N.; CHAMBERS, S.; JOHNSTON, R. Administração da Produção. 2a. ed. São Paulo: Editora Atlas, 2002.

SOOSAY, C. A.; HYLAND, P. W.; FERRER, M. Supply chain collaboration: capabilities for continuous innovation. Supply Chain Management, v. 13, n. 2, p. 160-169, 2008.

SQUIRE, B. et al. The effect of supplier manufacturing capabilities on buyer responsiveness: The role of collaboration. International Journal of Operations \& Production Management, v. 29, n. 8, p. 766-788, 2009.

THOMAS, R. W.; ESPER, T. L.; STANK, T. P. Testing the Negative Effects of Time Pressure in Retail Supply Chain Relationships. Journal of Retailing, v. 86, n. 4, p. 386-400, 2010.

VANDEGRIFT, D. Asset specificity, long-term contracts, and the good faith requirement. Eastern Economic Journal, v. 24, n. 4, p. 475-493, 1998.

VERGara, S. C. Projetos e Relatórios de Pesquisa em Administração. 3 ed. ed.São Paulo: Editora Atlas, 2000.

VILLENA, V. H.; REVILLA, E.; CHOI, T. Y. The dark side of buyer-supplier relationships: A social capital perspective. Journal of Operations Management, v. 29, n. 6, p. 561-576, 2011.

VIVALDINI, M.; PIRES, S. R. I.; SOUZA, F. B. Importância dos Fatores Não-tecnológicos na Implementação do CPFR. Revista de Administração Contemporânea - RAC, v. 14, n. 2, p. 289-309, 2010.

WIENGARTEN, F. et al. Collaborative supply chain practices and performance: exploring the key role of information quality. Supply Chain Management, v. 15, n. 6, p. 463-473, 2010.

YIN, R. K. Case study research: design and methods. 2. ed. Thousand Oaks: Sage Publications, 1994.

ZENZ, G. J. Purchasing and the Management of Materials. 7a. ed. EUA: John Wiley \& Sons, 1994.

ZSIDISIN, G. A.; ELLRAM, L. M. Activities related to purchasing and supply management involvement in supplier alliances. International Journal of Physical Distribution \& Logistics Management, v. 31, n. 9, p. 629-646, 2001. 